\title{
Safer services: conclusions from the report of the National Confidential Inquiry
}

\author{
Louis Appleby
}

In May 1999, the National Confidential Inquiry into Suicide and Homicide by People with Mental Illness published its first major report since it was reestablished at the University of Manchester (Appleby et al, 1999a). Entitled Safer Services, it presented findings from a two-year sample of suicides and an 18-month sample of homicides occurring in England and Wales, and on the basis of these put forward 31 recommendations for mental health services (Appleby, 1999b; Shaw et al, 1999). This article, modified from a chapter in Safer Services, discusses how services might deal with the risk of suicide and homicide or serious violence.

The recommendations are listed in the Appendix. We are aware that major intervention studies are needed to show conclusively that these measures will prevent suicide and homicide. However, in the absence of such evidence, we believe that the Inquiry findings indicate the components of good clinical practice. Most of the recommendations require agreed action by health authorities and hospital trusts, and the support of National Health Service (NHS) Executive regional offices. Some need specific action by the Department of Health or the Royal College of Psychiatrists.

\section{Preventability of suicide}

The Inquiry found that $24 \%$ (2370 of 10040 ) of suicides in England and Wales occurred in people who were in contact with mental health services in the year before death. This figure is an indication of the potential reduction in the population suicide rate that could be achieved by mental health services alone. For example, for mental health services to bring about a $15 \%$ reduction in the suicide rate, in line with The Health of the Nation (Department of Health, 1992), they would have to prevent over $60 \%$ of the suicide cases with whom they have been in recent contact - an unrealistic expectation in the short term. Suicide prevention requires a broadbased strategy incorporating population initiatives to reduce key risk factors, such as alcohol and drug misuse, and measures to reduce the availability or the lethality of common means of suicide, such as the detoxification of car exhaust emissions.

However, the figure of $24 \%$ corresponds to over 1000 suicides per year in close proximity to mental health care, and it is clear from our detailed findings that services could be made safer in a number of ways. It is a commonly held view in mental health services that suicides by people with mental disorder are often impossible to prevent because of the unpredictability of suicide and the high risk that is inherent in most disorders. Our data do not support this pessimistic view. Even the staff who provided us with information on their patients, who could be excused for underestimating, felt that the suicides were preventable in $22 \%$ of cases, that is, over 200 deaths per year. In two-thirds of cases, the staff were able to identify elements of service provision that would have made the suicide less likely - most often closer supervision of patients and improved patient compliance.

Louis Appleby is a Professor of Psychiatry at the University of Manchester and a consultant psychiatrists to the psychiatric Mother and Baby Unit at South Manchester University Hopsitals Trust. His principal research interests are suicide, self-harm and postnatal psychiatric disorders. His is Director of the National Confidential Inquiry into Suicide and Homicide by People with Mental Illness (School of Psychiatry and Behavioural Sciences, University of Manchester, Withington Hospital, Manchester M20 8LR; e-mail: Louis.Appleby@man.ac.uk). 
The Inquiry's findings suggest the need for substantial changes to the way in which mental health services currently operate. Some of these concern broad topics such as service priorities, training policy and information transfer; others concern specific aspects of clinical care. The Inquiry did not find, nor did it primarily set out to find, evidence of individual errors that contributed to particular suicides. Most mental health professionals work according to the structure and demands of their service in ways that reflect their service requirements and their own training. Our approach was, therefore, to relate suicides to the way in which services routinely work - to look for problems in the system rather than in the individual practitioner. We believe that, as a result, our conclusions and the recommendations based on them are generally applicable.

The finding that $24 \%$ of suicide cases were in contact with mental health services in the year before death raises important questions about the accessibility of services for the remainder, especially as several studies have shown that most people who commit suicide have a clinically significant mental disorder at the time (Barraclough et al, 1974; Foster et al, 1997; Appleby et al, 1999c). The Inquiry has not studied the factors that limit access but this is clearly an important subject for research. Limiting factors are likely to include the stigma of mental illness, public knowledge about mental illness, the recognition of risk in primary care, and the availability of crisis services.

The purpose of our report was to improve mental health services, and inevitably it highlights areas in which services are deficient in some way. It is, therefore, important to emphasise that the Inquiry also found considerable evidence of good practice in several areas of activity. Some of our recommendations are intended to bring all services in line with the best services. Others, however, are aimed at major change in the system as a whole.

\section{Preventability of homicide}

The Inquiry found that $8 \%$ (58 of 718 ) of people convicted of homicide had been in contact with mental health services in the year before the offence, and that $14 \%$ had been in contact at some time in the past. These figures, particularly the latter, may underestimate the true rate of contact because it was not possible for us to detect all service contacts that occurred many years ago, or in a part of the country far away from where a perpetrator was living at the time of the offence. This is because service contacts were identified from the records of mental health services in the perpetrator's district of residence, or neighbouring districts.

Nevertheless, the figures allow comparison with suicide. The rate of $8 \%$ in contact with services within a year corresponds to a little under 40 cases per year, whereas the equivalent figure for suicide is over 1000 cases, more than 25 times higher.

The Inquiry findings lead to additional conclusions about rates of mental disorder that are relevant to the prevention of homicide by health services. First, there was surprisingly little overlap between those who had been in recent contact with services and those who were found to have been mentally ill at the time of the offence. According to psychiatric court reports, only $20 \%$ of the latter had contact in the 12 months before the offence. Although this further emphasises the need to improve access to mental health services in some cases, it cannot be assumed that all cases should have been under mental health services. A large proportion of mental illness in the community is looked after in primary care, particularly less severe disorders including many cases of depression.

Second, in all homicides, as well as in the Inquiry sample (those with a history of mental health service contact), there was a striking prominence of alcohol and drug misuse. Of all people convicted of homicide, 39\% had a history of alcohol misuse, 35\% had a history of drug misuse, and $14 \%$ had a primary diagnosis of alcohol or drug dependence. Any public health strategy for preventing homicide would have to focus on alcohol and drugs at least as much as on mental illness.

Third, those who were under mental health care were likely to have a diagnosis of personality disorder $(19 \%)$, or alcohol or drug dependence $(31 \%)$, rather than severe mental illness. The term 'community care patients' is often used in discussing homicide and mental disorder to denote those patients who were, or should have been, under the care of a community mental health service before committing homicide. It therefore implies severe mental illness and/or considerable needs. Among Inquiry cases whose contact with services was within a year of the offence, around three-quarters had schizophrenia or depression, or had previously required hospital admission, regardless of the nature of their disorder. If these are regarded as homicides by community care patients, the number of such cases is around 25 per year. This is a small figure as a proportion of all homicides, and represents a tiny fraction of all those who are treated by community mental health services, but it is not insignificant. The critical question for services is the extent to which these tragic incidents are preventable.

The Inquiry findings show both the limits of and the potential for prevention. Compared to suicides, 
homicides did not occur in such close proximity to services - relatively few convicted patients had been in contact with staff in the week before the offence. More of the homicide perpetrators were suffering from disorders that are regarded as difficult to treat, for example, personality disorder. The mental health teams who gave us information rarely believed that the homicide had been preventable. However, they did in half the cases indicate measures that would have made the homicide less likely - once again, improved patient compliance was most often suggested. Moreover, our detailed findings on contact with services make clear several ways in which services need to be strengthened. As these are generally comparable to the needs for suicide prevention, discussions of suicide and homicide are combined in the rest of this article.

\section{Priority groups of patients}

We believe that there should be no higher priority in planning care than the risk of suicide and serious violence, that risk should rank beside the other main priorities such as social needs and that the breadth and intensity of services that patients receive should be proportionate to the risk that they present to themselves and others.

Our findings show that a large number of patients who commit suicide or homicide, including many with severe mental illness, are not subject to the higher levels of the Care Programme Approach (CPA). Many patients who commit suicide have been thought to need less intensive service support soon after their acute illnesses have subsided, even though many of their risk factors are unchanged. Our findings highlight the main characteristics of patients who commit suicide and show how often certain risk factors for suicide, identified in previous studies, are found. We are not yet at the stage of drawing up a questionnaire that can be used to estimate the degree of risk in an individual patient based on the presence of combinations of key risk factors, although this is a future aim. However, it is possible from our data to describe groups of patients in whom a large proportion of all suicides under mental health care will occur. The main indicators of risk that were presented are deliberate self-harm, alcohol or drug misuse, and a history of hospital admission; other risk indicators include detention under the Mental Health Act, comorbidity and social isolation.

Our findings also show that a majority of patients who kill have previously been violent, particularly in the year before the homicide. They have high rates of alcohol and drug misuse and deliberate self-harm. They have frequently been admitted to hospital at some time, often under the Mental Health Act. Those with severe mental illness often have secondary diagnoses. Many of these risk indicators are common to both suicide and homicide.

Using such factors, particularly when they occur in combination, a service can identify a group of patients who should be the priority for its preventive activities, not only at times of acute illness but also in the long term. To do this effectively, a service needs to record key indicators of risk when a patient first makes contact with a service and to review these at regular intervals. Its documentation needs to be designed to reflect these priorities.

In concentrating its activities on priority groups based on estimates of risk, a service will have to accept that it will be providing more intensive care to many patients who would not in any case commit suicide or serious violence. This is sometimes seen as wasteful of resources, but we believe it can be justified because, in the relative absence of specific suicide and homicide prevention measures, the activities that are required - closer supervision, maintenance of treatments, etc. - are in fact aspects of high-quality care. The primary aim of a properly focused service may then be to reduce risk, but the secondary benefit is that many of the most needy patients will receive a better service.

It is also important to recognise that greater service activity for one group of patients must mean doing less of something else. At present, it is apparent that mental health staff spend too much time on administrative aspects of care, as well as on routine reviews of patient care that do not differ from one occasion to the next and often do not include the patient or his or her family. Mental health services should ensure that:

- contacts between patients and services always serve a specific agreed purpose, and in most cases this includes assessment of risk;

- health professionals do not duplicate each other's work; and

- patients without severe mental illness are considered for referral to a less specialised service, such as primary care, if they have no major indicators of risk and no specific acute treatment needs that justify remaining in the secondary service.

Routine appointments, serving no definite purpose, should become a thing of the past in a specialised service.

If the risk of individual patients is a major determinant of how resources are allocated within a service, then the risk-related characteristics of a patient population should be a major determinant 
of how resources are allocated to a service. Where there are high rates of comorbidity or substance misuse, these should be clearly reflected in funding. Services should therefore collect risk-related information; some information, such as the percentage of staff receiving training, should be used to assess service performance in relation to risk (Recommendation 7).

\section{Recommendations}

\section{Training}

We believe that to operate more safely, services need to improve skills rather than increase paperwork. There has been a large expansion of the latter in recent years; what is now required is a programme of training in the recognition, assessment and management of risk (Recommendations 1 and 2).

Our findings show that $85 \%$ of suicides occur in patients who are regarded as being at low immediate risk at the final contact with services, even though this frequently takes place within a week before death and in many cases within 24 hours. This does not mean that services assess risk poorly, because studies of this kind do not include people whose risk is recognised and who are then successfully treated. Nor does it mean that high risk was missed in these cases, because risk may vary as circumstances change. However, it suggests that, at least in some suicides, there are opportunities for prevention, particularly when contact has been recent, and it implies that in some cases this could be achieved by more accurate risk assessment.

Even so, the finding that many suicide and homicide cases are thought to be at low immediate risk at final service contact is not in itself evidence of the need for widespread training. Several other findings in this report point to that conclusion. For example, our data show that suicide risk assessment in clinical practice relies most heavily on abnormalities of current mental state and suicidal ideas. This is understandable when these are present, but misleading when they are absent, as they frequently are. Many of the Inquiry suicides who were thought to be at low immediate risk carried a number of risk factors at all times and could have been regarded as at constant high risk. In the three months before suicide, many who showed no direct evidence of clinical relapse indicated their risk in less direct ways, for example, through increased alcohol or drug misuse, or non-compliance with treatment. However, these 'proxy indicators' of relapse did not lead to an awareness of increasing risk.
In our homicide data, the pattern of contact was similar. Although the homicides were less likely to have been in contact with services in the recent past, almost a quarter of those who were under mental health care in the year before the homicide were assessed in the week before the offence. Around a third of final contacts were non-routine. At final contact, few expressed violent ideas - increased alcohol or drug misuse was more common. In $94 \%$ of cases, risk was estimated to be low.

Training in risk management should aim to make full risk assessment, using all relevant evidence, a routine part of clinical practice for all 'front-line' staff - those in contact with high-risk patients at high-risk times, including both community and ward staff. Although most people assessing the Inquiry suicide cases at final contact had had some form of previous training in suicide risk assessment, only around half of the staff at the final contact with the homicide cases had had training in the assessment of risk of violence. Assessing risk of violence is sometimes seen as the role of forensic mental health services, but most of the Inquiry homicide cases were committed by people under general psychiatric services.

When we surveyed trusts nationally, we found that only half offered their nursing staff training in assessment of either suicide or violence risk. This is an unacceptable situation in which staff do not appear to have the opportunity for regular improvement in skills that are central to the safety of patients, families and the public. All health regions have a training budget, now devolved to regional educational training consortia, which should develop training programmes in risk assessment and management.

The need for regular training and routine assessment of risk applies to all services that care for highrisk patients, including those that are not now part of mainstream mental health care. We were concerned to hear regularly that alcohol and drug teams do not assess risk because they do not regard it as their responsibility - an untenable and unsafe argument.

\section{Documentation/information}

The need to record all relevant risk factors for suicide and serious violence at entry to a service, and to review these at regular intervals, has already been emphasised. Accurate assessment also requires proper documentation, and multi-disciplinary management of risk requires the transfer of relevant information between individual professionals and between agencies.

One of the most frequent recommendations in previous reports on mental health services was for 
better communication between staff. In the Inquiry cases, once moderate or high risk had been identified, this information was usually, although not always, passed on to other staff, but in some cases by writing in the case notes. Yet, it is common practice in mental health for each professional discipline to keep its own set of notes in a way that is never available to the others (see Recommendation 4).

Our findings show that when patients attend new services, key information relevant to their risk of suicide often does not accompany them. The solution to this dangerous practice will lie in developments in information technology, leading to shared electronic case notes. In the interim, services need to develop their paper case notes to ensure structured recording of information and sharing of relevant information between disciplines within a service, and to adopt protocols for the transfer of information to other agencies and to services in other districts.

We do not want to add to the list of reports that have made a general call for better communication, but to prompt specific developments in the recording and use of information concerning risk of suicide and serious violence. We are therefore proposing a single, simplified but universal system of documentation, 'patient passports', which will serve three related purposes (Recommendation 3):

- clinical risk assessment, by the recording of key indicators of risk;

- allocation to care under the CPA according to evidence of risk, and subsequent monitoring; and

- transfer of information between services.

As a first step, the Department of Health should identify examples of good practice in local CPA documentation.

In the homicide cases, one of the most notable findings was that previous convictions for violence were frequently not recorded in case notes. Such information is vital to risk assessment. Our data provide a strong argument for making information on convictions, currently held by the police, available to mental health services on request (Recommendation 6). Although this could give rise to concerns over confidentiality and privacy, we believe these are outweighed by the need to improve safety.

\section{Care Programme Approach}

The CPA, the centrepiece of mental health service provision in the community (Department of Health, 1990; Welsh Office, 1996), is in need of overhaul. This is not to say that it does not work well in many cases. It is clear from our data that it is applied to more severely ill patients and that for most of those whom it covers, it does what it is supposed to, providing a keyworker and ensuring regular review.

However, there are four serious problems with the CPA. First, it is not applied sufficiently to patients at risk. At its higher levels, intended to provide structured multi-disciplinary care for the most needy patients, it encompasses a minority of suicide cases and few patients who commit homicide. Even in patients with schizophrenia who commit suicide or homicide, perhaps the most obvious groups to be under the highest level of the CPA, it covers only two-thirds of cases. Priority for allocation to highlevel CPA should be given to all patients with severe mental illness who fall into the high-risk groups outlined above, and in particular to those with a history of violence (Recommendation 5).

Second, it does not prevent non-compliance with treatment or loss of contact with services, as findings from both the homicide and suicide cases make clear. It needs to be backed by stronger clinical and legal measures, as outlined below. It could be argued that the Supervision Register and supervised discharge provide the necessary backing already, but the Register is an administrative rather than a clinical measure and is not popular with health staff, and supervised discharge does not in itself allow treatment. Our sample included patients who lost contact with services despite being on the Supervision Register or under supervised discharge.

Third, it generally does not take account of changes in suicide risk over time. At the point of hospital discharge, therefore, long-term after-care is planned and formalised in the CPA, as if needs were uniform over the following months or longer. In fact, suicide risk is much greater in the first few months after discharge and service provision must reflect this period of maximum need (Recommendation 24).

Fourth, the documentation that the CPA requires is poorly regarded by clinical staff, and this is likely to explain those cases in which the CPA is implemented but review dates are unrecorded. Its clinical value is in danger of being undermined by its administrative demands. Assessment for the CPA is often separate from clinical assessment, and the two must be combined in an integrated information system. At its simplest level, and while developments in information technology are awaited, CPA documentation should be universally redesigned to be compatible with clinical assessment in general and risk assessment in particular, and should become part of case notes, rather than being a separate system (Recommendation 3 ).

\section{Treatments and non-compliance}

Non-compliance with treatment runs throughout mental health services and is a feature of at least a 
quarter of suicides and homicides. Addressing this problem is a matter of urgency.

It is important to stress that 'non-compliance' is a shorthand but slightly loaded term, implying reluctance on the part of the patient to do what staff believe is needed. In clinical practice - and in this article-non-compliance covers non-receipt of treatment for any one of a number of reasons. It is a reflection of the broader relationship between patient and service, and solving the widespread problem of noncompliance requires an understanding of the reasons behind it. Our focus, however, has been narrower than this, and concerns the non-receipt of potentially effective treatment by people who are at risk.

There is an absence of policy on non-compliance in most district services. In many Inquiry suicides and homicides, possible interventions - including the use of modern antipsychotic and antidepressant drugs, motivational interviewing, and the involvement of families in ensuring treatment - do not appear to have been pursued. In high-risk cases with severe mental illness, intervention is essential. In a safe service, it should not be possible for high-risk people to be non-compliant or incompletely recovered while alternative treatments are untried, including family and cognitive interventions.

However, at present, the more modern drug treatments can only be taken orally, which makes compliance more difficult to monitor. It is also unrealistic to expect services to make family and cognitive interventions available to large numbers of patients in the short term, because they require trained staff to deliver them. Nevertheless, these nonpharmacological treatments should be available at least for those at high risk (Recommendation 9). Newer drug treatments are more readily available to services and, despite their cost, should be used in severe mental illness where older antipsychotic drugs cause side-effects that prevent compliance (Recommendation 8). Ultimately, in some cases, appropriate mental health legislation is needed (see below).

One surprising feature of drug treatments in our sample was the frequent use of benzodiazepines to treat anxiety in patients with severe mental illness, including schizophrenia. Our concern is that in at least some of these patients, anxiety is an indicator of relapse of major illness, requiring a review of clinical management as a whole rather than symptomatic treatment.

\section{Disengaged patients}

Non-attendance and loss of contact with services frequently precede suicides and homicides. Nonattendance was reported in a quarter of both suicides and homicides; loss of contact had occurred in no fewer than $71 \%$ of homicides. Reducing the problem of disengagement is, as with non-compliance to which it is closely related, a service priority.

Disengaged patients are a mixed group but include:

- patients with severe mental illness who appear to be disengaged from society as a whole (living alone, unmarried, unemployed) as well as from clinical services;

- patients at risk who lose contact with services; and

- homeless patients.

The first of these, more than any group, requires broad packages of care, incorporating social support, daily care facilities, assertive outreach and occupational activities, and therefore multi-professional coordination (Recommendation 11). They require close observation after hospital admission, when many commit suicide following absconding. At present, only a minority of services has a policy for this needy group.

Suicide cases among homeless people have similar characteristics and needs. Although there is good evidence for the benefits of assertive community treatment (Marshall et al, 1996), including in the care of homeless people with mental illnesses (Dixon et $a l, 1997)$, its development has been patchy (Recommendation 12).

Our data show that in many cases of suicide and homicide, including cases of severe mental illness, assertive actions to re-engage patients, such as home visits, are not taken. In a safe service, it should not be possible for people with severe mental illness who are at high risk of suicide or serious violence to drop out of care without strenuous efforts being made to maintain contact, ultimately including the use of mental health legislation (see below).

\section{Comorbidity}

Throughout our findings, there is repeated evidence that alcohol and drug misuse are among the main problems facing the development of safer services. Both suicide and homicide cases have high rates of alcohol and drug dependence and misuse. Increased alcohol and drug misuse frequently occurs in the period leading to suicide or homicide, often in the absence of more direct indications of clinical relapse. Yet, for a variety of reasons, specialist services for alcohol and drugs are now often separate from mainstream mental health services, many patients fall between the two services, and the capacity of general psychiatry services to address these problems falls well short of the clinical need. 
Despite the absence (so far) of clear evidence for the effectiveness of any intervention for patients with both severe mental illness and substance misuse (Weaver et al, 1999), two measures can be regarded as essential in equipping services for the provision of treatment of alcohol and drug misuse as an integral part of mental health services (Recommendation 14) - the problem of substance misuse is now in a central position in mental health services and cannot continue to be the domain of a distant speciality. First, staff numbers need to be sufficient - the number of cases of comorbidity in a service should be a determinant of health authority spending on mental health (Recommendation 7). Second, staff need to be trained to provide clinical management (Recommendation 15).

\section{Ethnic minorities}

Our findings do not suggest the need for specific measures to reduce suicide in ethnic minority patients because, in general, their characteristics were similar to those of the sample as a whole. However, some of the measures required for all patients at risk are particularly needed by those from ethnic minority groups. This is especially true of initiatives to reduce non-compliance with drug treatment, which was more common in ethnic minority patients in the three months before suicide.

\section{Contacts with families}

Care of people with mental illness is shared with their families, but when crises arise, families who want to alert services can encounter difficulties in gaining access. We plotted the sequence of events prior to suicide and found that clinical contact with relatives tended to occur late, if at all. In two homicide cases, the final contact with services was arranged at the request of families. It is vital that families are treated as partners in the care of people at risk so that the information they have can quickly be passed on directly to the professionals. They need a direct and easy route to the mental health team providing care for their relative - one that does not involve negotiating their way through switchboards or leaving messages with unknown staff (Recommendation 16).

Information provided to families should include routes of access in times of crisis. Similarly, families are often aware of non-compliance and concerned by loss of contact, and they should also be given information on policies in these areas (Recommendations 10 and 13).
When a suicide or a homicide has occurred, families need information, provided openly and promptly (Recommendation 28). Many services appear already to hold discussions with families after suicide, but this is unusual after homicide. The offer of information in these circumstances should become routine practice for all services.

\section{In-patient suicides}

Suicides by current in-patients are seen as the most preventable group in our case series. The numbers are substantial - 358 in two years, $16 \%$ of our sample, and $4 \%$ of all suicides in the population. These figures show that in-patient suicides are more than twice as frequent as prison suicides over which there is (correctly) great public concern.

Five problems need to be addressed. First, twothirds of suicides occurring on wards are by hanging. All wards need to remove the structures that allow this to happen, including non-collapsible curtain rails, door handles and / or coat-hooks (Recommendation 17). Second, a quarter of suicides occur on wards in which there are structural difficulties in observing patients. These wards are unsuitable for the admission of acutely ill patients (Recommendation 17). Third, many in-patients who commit suicide are allowed off the ward by staff believing them to be well. Training in risk assessment for all ward staff and greater caution in granting leave during recovery are needed (Recommendation 20). Fourth, suicides on the ward itself cluster in the evening and at night-more intensive observation at this time is needed (Recommendation 19). Fifth, our findings raise serious doubts about the value of current observation protocols. To our knowledge there has never been a clinical trial of the kind of observation practices that are in widespread use. It also seems that there is great variation nationally in the observation of patients at risk. Suicides by patients under an intermediate level of observation (e.g. where the patient is to be observed every 10-15 minutes) are not uncommon. Whether observation at intervals of this kind are of any value in preventing suicide, selfharm or absconding must be in doubt. Trusts should consider the criteria by which patients are moved from intensive to intermediate observation levels, and work to develop alternative models of observation, for example, constant observation of defined areas of a ward (Recommendation 18).

\section{Post-discharge suicides}

The immediate post-discharge clustering of suicides is one of the greatest priorities for prevention. 
Over two years, 519 suicides were in this group $24 \%$ of the Inquiry sample. Our findings make clear that patients fall between in-patient and community care at this critical transition. The largest weekly number of suicides was in the first week after discharge. Forty per cent of all postdischarge suicides occurred before the first followup appointment.

We strongly support better integration of inpatient and community teams and their activities (Recommendation 23). This requires immediate follow-up for all high-risk patients, including many whose admission has been brief, and rapid followup for all discharges (Recommendation 21). In particular, we are extremely concerned about the practice of admitting acutely ill patients to hospitals in other districts, which usually happens because of shortage of suitable acute facilities locally (Recommendation 22). The consequence is that these patients are discharged to community services that may be distant from where they have received inpatient care, and fully coordinated transfer of care to the community becomes almost impossible. This is obviously an inhumane practice because it can take patients far from their homes and families. Our finding that post-discharge suicides begin - in fact, are most likely - immediately after leaving hospital suggests that it is also dangerous.

\section{Mental Health Act 1983}

The Mental Health Act has recently been under review. Our findings support the need for treatment to be maintained in patients who would be at substantial risk of suicide or violence if they were to relapse. It is clearly unacceptable that patients who have a history of violence, or serious aggressive behaviour, in the context of mental illness should be allowed to be non-compliant with any effective treatment or to lose contact with services, as occurred in a majority of cases prior to homicide. The same is also true of patients at high risk of suicide.

In these patients, it is not safe to wait until risk has clearly manifested itself before turning to the use of the Act. It should be possible to treat highrisk patients as soon as there are clear signs that a familiar sequence of events has begun that will lead to suicide or serious violence. The Mental Health Act Commission (1998) has clarified that the Act can be used before relapse has become severe. Our data show that clinical relapse in the sense of mental state symptoms frequently does not occur prior to suicide and homicide, but that many patients show 'proxy indicators', including simple noncompliance with treatment. The new Act must allow treatment in the community at the earliest point in cases of high risk (Recommendation 25).

However, this extension of its powers increases the need for more skilled assessments and suitable training. These too should become a requirement of the Act, placed on the professionals who will use it. It also needs to be emphasised that the proportion of suicides and homicides that will be prevented by enforced community treatment is small. If, for example, the target of such treatment were people with schizophrenia or affective disorder who in their last admission had to be detained under the Mental Health Act, and if all suicides and homicides were prevented in which non-compliance or nonattendance had recently occurred, then according to our data, 30 suicides and two homicides would be prevented per year.

\section{Access to means of suicide}

Most suicides under mental health care take place by hanging or overdose with psychotropic drugs. These are the methods that must be addressed by measures designed to reduce access to the means of suicide.

There is a clear need to restrict access to the more toxic psychotropic drugs, through the use of alternative treatments, for example, modern antidepressants, or short-term supplies (Recommendation 26). When patients are at risk, services should first ensure that they do not provide the means of suicide themselves. Our data show that, at present, patients known to have a history of self-harm are more likely to commit suicide by overdose of the drugs used in their treatment.

Hanging is a much more difficult method to restrict, but its frequent use in in-patient suicides in whom it is the method in $40 \%$ of cases emphasises the need for the physical structure of wards to be reviewed (see Recommendation 17).

\section{Aftermath of suicide and homicide}

A majority of services already reviews suicides, and some review homicides, in a multi-disciplinary forum. This is an example of good practice that should be universal (Recommendation 27). Making information available to families is discussed above (and see Recommendations 10 and 13).

\section{Personality disorder}

It was not the purpose of the report to make detailed recommendations on the treatment of patients with personality disorder. However, there are 
numerous references to personality disorder in our findings and it is important to summarise what they show.

Patients with personality disorder are the largest diagnostic group in the homicide cases (22\%) and one of the larger groups in the suicides $(11 \%)$. In both parts of the Inquiry, patients with personality disorder had high rates of risk factors, including previous violence, but had highly disrupted patterns of care, with frequent noncompliance and loss of contact. In the suicide cases, those with personality disorder were seen as the least preventable.

It is clear that mental health services are not currently able to provide satisfactory care to this group. They do not fall within any definition of severe mental illness on which priority in service provision, such as the CPA, is based. There is no good evidence on which to base treatment; as a result they cannot be detained under the 'treatment section' of the present Mental Health Act. Nevertheless, services can and should provide support and supervision for people with personality disorder at times when they are at risk of suicide, as they do with other groups.

The greater problem is in preventing violent behaviour. Likelihood of violence is in itself not a reason to receive treatment from mental health services, but, in many cases, the diagnosis of personality disorder indicates little else. As a result of the diagnosis, some violent patients fall between the two stools of mental health services and the criminal justice system, and part of the solution to the problems posed by patients with violent personality disorder is to locate them more firmly within one or the other (Recommendation 29).

One alternative is, therefore, to bring patients with violent personality disorder more clearly under mental health services, which would have to become more custodial - a solution that would be unacceptable to many staff, patients and families. The other is to bring them more clearly into the criminal justice system, which would have to become more therapeutic - a solution with the potential for broader benefits to criminal recidivism.

\section{Stigma}

One of the concerns of patient groups and others is the preoccupation in the press and elsewhere with violence committed by people with mental illness and the risk that such people pose to the general public. Critics of such publicity argue that most serious violence is not committed by people with mental illnesses, that the great majority of people with mental illnesses are not violent and that to focus on rare incidents of serious violence adds to the stigma that limits the rights and freedoms of people with mental illnesses.

Our findings offer support to these views. Homicides by people with severe mental illness are few; when they do occur, it is patients' families rather than the public at large who are usually the victims; many mentally ill perpetrators are not, and may never have been, under mental health services.

However, this article also makes clear that there are numerous ways in which services could and should be made safer. The data collected by the Inquiry should be used by the Royal College of Psychiatrists, which has been conducting an antistigma campaign (Royal College of Psychiatrists, 1998), to present a balanced view of this issue to the press and public, for example, when violence by someone with a mental disorder receives publicity (Recommendation 30).

\section{'Culture of blame'}

Unsolicited comments from staff who completed our questionnaires showed a strong and widely held view in mental health services that when a homicide or other serious untoward incident occurs, staff will be unfairly blamed. This is particularly how the mandatory local inquiries into individual homicides are viewed. As a result, local inquiries cannot provide the best setting for lessons to be learned.

We believe that the National Confidential Inquiry now serves this purpose. Cooperation from mental health staff is excellent. Information is provided without fear of blame. Data from a substantial number of cases are aggregated for analysis; as a result, conclusions are likely to be generalisable to services nationwide - inquiries into individual cases may have narrow relevance.

Where then does this leave local inquiries? In theory they are capable of serving several purposes, for example: information for relatives of patients and/or victims; public reassurance about the accountability of public services; highlighting of the need for improvements in services; and identification of negligence. In practice, their benefits are not so clear, but their cost is high. We proposed in our report that it was time to reassess the purpose and value of local inquiries, and that the Department of Health should consider alternatives to the present system (Recommendation 31). Subsequently, the Department commissioned the Health Advisory Service 2000 to draw up a new inquiry system for individual adverse incidents; its report is awaited. 


\section{Appendix: Recommendations in Safer Services}

\section{Training}

1. All staff in contact with patients at risk of suicide should receive training in the recognition, assessment and management of risk, of both suicide and violence, at intervals of no more than three years.

2. The content of training should reflect many of the points highlighted by this report: indicators of risk, high-risk periods, managing noncompliance and loss of contact, communication, and the Mental Health Act.

\section{Documentation/information}

3. A new, simplified, universal system of documentation (patient passports) should be developed, to be used for three purposes:

- clinical risk assessment, by the recording of key indicators of risk;

- allocation to care under the CPA according to evidence of risk, and subsequent monitoring; and

- transfer of information between services.

4. Unified systems of case notes for all professional disciplines should be developed.

5. All patients with a history of violence in the context of mental illness should receive the highest level of care under the CPA.

6. Information on previous convictions for violent offences should be readily available to mental health services on request.

7. Risk-related information, e.g. rates of comorbidity and staff training, should be collected and used in determining resources and monitoring performance.

\section{Treatments and non-compliance}

8. Modern drug treatments such as 'atypical' antipsychotic drugs and newer antidepressants should be offered to all patients with severe mental illness who are noncompliant with treatment because of sideeffects.
9. Family and psychological interventions should be available to all high-risk patients with severe mental illness.

10. Trusts should have a written policy on noncompliance, based on these recommendations, which is made known to staff, patients and families.

\section{Disengaged patients}

11. In all patients with severe mental illness who have a history of disengagement from services, a comprehensive social and clinical care plan should be devised that includes satisfactory housing and occupational activities.

12. Services should have the capacity for assertive outreach in response to loss of contact with patients with severe mental illness, including those who are homeless.

13. These recommendations should be part of a written policy on disengagement, which should be made known to staff, patients and families.

\section{Comorbidity}

14. Services should make provision for patients with severe mental illness and alcohol or drug misuse as part of mainstream mental health services.

15. Training of staff in general psychiatry services should include the management of alcohol and drug misuse.

\section{Families}

16. 'Points of access' to mental health teams should be provided for families who are concerned about a patient's risk.

\section{In-patient suicides}

17. All services should review the physical structure of wards to identify:

- any obstructions to the observation of highrisk patients; and

- structures that could be used in suicide by hanging. Wards in which these cannot be removed should not be used for the admission of acutely ill patients.

18. Alternatives to intermediate level observations should be developed for patients at risk. 
19. Services should increase and monitor the observation of patients in the evening and at night.

20. Risk assessment should always be carried out prior to granting leave in patients who are recovering from illness.

\section{Post-discharge suicides}

21. There should be follow-up within 48 hours for all patients who have been at high risk and who are discharged from in-patient care, and follow-up within one week for all discharges, including those who discharge themselves.

22. Health authorities and trusts should make provision to accommodate all acutely ill patients in local catchment area services, ending transfers to in-patient care in other districts.

23. Prior to discharge from in-patient care, inpatient and community teams should conduct a joint case review, including assessment of risk.

24. CPA documentation should include more intensive provisions for the first three months after discharge from in-patient care, and specific reference to the first post-discharge week.

\section{Mental Health Act}

25. Mental health legislation should allow the enforced treatment of high-risk patients with severe mental illness who become noncompliant with treatment or who show indications of increasing risk, even in the absence of clear signs of relapse.

\section{Access to means of suicide}

26. Patients at risk of suicide, including all patients with a recent history of self-harm, who are treated with psychotropic drugs should receive modern, less toxic drugs and/or supplies lasting no more than two weeks.

\section{Aftermath of suicide or homicide}

27. Following a suicide or a homicide, mental health teams should hold a multi-disciplinary review of the case.

28. Following a suicide or homicide, information on what happened should be provided promptly and openly to families.

\section{Personality disorder}

29. Clear policies on the clinical management of personality disorder should be disseminated by the Department of Health.

\section{Stigma}

30. Information in this report should be used by the Royal College of Psychiatrists to inform the public on the risks posed by people with severe mental illness, both to themselves and others.

\section{Culture of blame}

31. The Department of Health should assess the purpose and value of local inquiries into serious untoward incidents, and consider changes to the current requirement for full-scale inquiries in all cases.

\section{References}

Appleby, L., Shaw, J., Amos, T., et al (1999a) Safer Services. Report of the National Confidential Inquiry into Suicide and Homicide by People with Mental Illness. London: Department of Health.

_- _- _- et al (1999b) Suicide within 12 months of contact with mental health services: national clinical survey. British Medical Journal, 318, 1235-1239.

-, Cooper, J., Amos, T., et al (1999c) Psychological autopsy study of suicides by people under 35. British Journal of Psychiatry, 175, 168-174.

Barraclough, B. M., Bunch, J., Nelson, B., et al (1974) A hundred cases of suicide; clinical aspects. British Journal of Psychiatry, 125, 355-373.

Department of Health (1990) Caring for People. Care Programme Approach for People with a Mental Illness referred to the Special Psychiatric Services. London: HMSO.

- (1992) The Health of the Nation. London: HMSO.

- (1994) Guidance on the Discharge of Mentally Disordered People and their Continuing Care in the Community. HSG (94)27. London: NHS Executive.

Dixon, L., Weiden, P., Torres, M., et al (1997) Assertive community treatment and medication compliance in the homeless mentally ill. American Journal of Psychiatry, 154, 1302-1304.

Foster, T., Gillespie, K., McClelland, R. (1997) Mental disorders and suicide in Northern Ireland. British Journal of Psychiatry, 170, 447-452.

Marshall, M., Gray, A., Green, R. (1996) Case Management for People with Severe Mental Disorders In Cochrane Library, Issue 3. Oxford: Update Software.

Mental Health Act Commission (1998) The Threshold for Admission and the Relapsing Patient. Nottingham: Mental Health Act Commission.

Royal College of Psychiatrists (1998) Changing Minds: every family in the land. Psychiatric Bulletin, 22, 328-329.

Shaw, J., Appleby, L., Amos, T., et al (1999) Mental disorder and clinical care in people convicted of homicide: national clinical survey. British Medical Journal, 318, 1240-1244.

Weaver, T., Renton, A., Stimson, G., et al (1999) Severe mental illness and substance misuse. British Medical Journal, 318, 137-138.

Welsh Office (1996) Guidance on the Care of People in the Community with a Mental Illness. Cardiff: Welsh Office. 